\title{
Pressure tuning of the anomalous Hall effect in the chiral antiferromagnet $\mathrm{Mn}_{3} \mathrm{Ge}$
}

\author{
R. D. dos Reis $\odot,{ }^{1,2}$ M. Ghorbani Zavareh,,${ }^{1, *}$ M. O. Ajeesh, ${ }^{1}$ L. O. Kutelak $\odot,{ }^{2}$ A. S. Sukhanov, ${ }^{1}$ Sanjay Singh, ${ }^{1,3}$ J. Noky, ${ }^{1}$ \\ Y. Sun, ${ }^{1}$ J. E. Fischer $\odot,{ }^{1}$ K. Manna, ${ }^{1}$ C. Felser, ${ }^{1}$ and M. Nicklas $\oplus^{1, \dagger}$ \\ ${ }^{1}$ Max Planck Institute for Chemical Physics of Solids, Nöthnitzer Strasse 40, 01187 Dresden, Germany \\ ${ }^{2}$ Brazilian Synchrotron Light Laboratory (LNLS), Brazilian Center for Research in Energy and Materials (CNPEM), \\ Campinas, Sao Paulo, Brazil \\ ${ }^{3}$ School of Materials Science and Technology, Indian Institute of Technology (BHU), Varanasi-221005, India
}

(Received 29 January 2020; revised manuscript received 7 April 2020; accepted 17 April 2020; published 7 May 2020)

\begin{abstract}
We report on the pressure evolution of the giant anomalous Hall effect (AHE) in the chiral antiferromagnet $\mathrm{Mn}_{3} \mathrm{Ge}$. The AHE originating from the nonvanishing Berry curvature in $\mathrm{Mn}_{3} \mathrm{Ge}$ can be continuously tuned by application of hydrostatic pressure. At room temperature, the Hall signal changes sign as a function of pressure and vanishes completely at $p=1.53 \mathrm{GPa}$. Even though the Hall conductivity changes sign upon increasing pressure, the room-temperature saturation value of $23 \Omega^{-1} \mathrm{~cm}^{-1}$ at $2.85 \mathrm{GPa}$ is remarkably high and comparable to the saturation value at ambient pressure of about $40 \Omega^{-1} \mathrm{~cm}^{-1}$. The change in the Hall conductivity can be directly linked to a gradual change of the size of the in-plane components of the Mn moments in the noncollinear triangular magnetic structure. Our findings, therefore, provide a route for tuning of the AHE in the chiral antiferromagnetic $\mathrm{Mn}_{3} \mathrm{Ge}$.
\end{abstract}

DOI: 10.1103/PhysRevMaterials.4.051401

The search for effective control and manipulation of spin degrees of freedom in solid-state systems has attracted huge attention in the past three decades due the potential for applications [1-6]. So far most spintronic devices are based on ferromagnetic materials. Only recently, antiferromagnetic materials came into focus due to many new phenomena, such as the large anomalous Hall effect (AHE), spin Hall magnetoresistance, skyrmions, and the spin Seebeck effect, enriching the microcosmic physics system and encouraging vigorous development of the new field of antiferromagnetic spintronics [7-11]. This field aims to complement or replace ferromagnets in the active components of spintronic devices. Compared to ferromagnets, antiferromagnetic materials resist perturbation well, producing no stray fields that perturb the neighboring cells. The latter removes an obstacle for high-density memory integration. Furthermore, they demonstrate ultrafast dynamics and generate large magnetotransport effects. In that sense, antiferromagnetic materials have become increasingly important and exhibit various promising applications, including nonvolatile memory and magnetic field probes. However, although several schemes have been theoretically proposed to achieve full control of an antiferromagnetic structure, the

\footnotetext{
*Present address: HC DI CT R\&D CTC SA, Siemens Healthcare GmbH, Siemensstrasse 3, 91301 Forchheim, Germany.

†michael.nicklas@cpfs.mpg.de

Published by the American Physical Society under the terms of the Creative Commons Attribution 4.0 International license. Further distribution of this work must maintain attribution to the author(s) and the published article's title, journal citation, and DOI. Open access publication funded by the Max Planck Society.
}

experimental demonstration is still challenging and remains elusive [12-14]. Therefore, finding ways to efficiently manipulate the magnetic states in antiferromagnets is the key to the further development.

The Heusler compound $\mathrm{Mn}_{3} \mathrm{Ge}$ is known to crystallize in both hexagonal and tetragonal structures [15]. While the tetragonal material presents ferrimagnetic ordering below the Curie temperature of $710 \mathrm{~K}$, the hexagonal exhibits a triangular antiferromagnetic structure with an ordering temperature of 365-400 K [16]. In the hexagonal structure, the unit cell of $\mathrm{Mn}_{3} \mathrm{Ge}$ consists of two layers of $\mathrm{Mn}$ stacked along the [0001] axis. In each layer, the $\mathrm{Mn}$ atoms form a kagome lattice, with Ge sitting at the center of a hexagon $[8,16,17]$. Previous neutron diffraction experiments characterize the spin arrangement as a noncollinear triangular antiferromagnetic lattice of the Mn spins in which neighboring moments are aligned in the (0001) plane at an angle of $120^{\circ}$ [18-21]. In this configuration the spin triangle is free to rotate in an external magnetic field, and due the geometrical frustration of the Mn moments, a weak ferromagnetism is observed along the [0001] axis.

Theoretical predictions demonstrate that this noncollinear triangular antiferromagnetic structure, common to all $\mathrm{Mn}_{3} X$ ( $X=\mathrm{Ge}, \mathrm{Sn}, \mathrm{Ga}$, Ir, $\mathrm{Rh}$, and $\mathrm{Pt}$ ) compounds, gives origin to a nonvanishing Berry curvature which was predicted to lead to a large AHE [15,22-27]. Indeed, Nayak et al. [8] reported a large AHE effect comparable to that observed for ferromagnetic metals even at room temperature $[5,28,29]$. Supported by the angular dependence of the AHE, they demonstrated that the small residual in-plane magnetic moment plays no role in the observed effect except to control the chirality of the triangular spin structure [see Fig. 1(a)]. Thus, they concluded that the large anomalous Hall effect in $\mathrm{Mn}_{3} \mathrm{Ge}$ originates from 
(a)
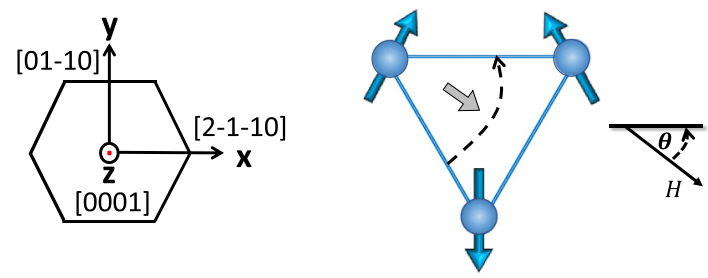

(b)

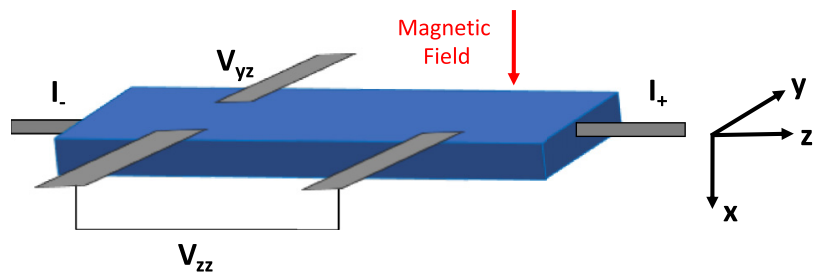

FIG. 1. (a) Illustration of the rotation of the triangular spin structure upon application of an in-plane magnetic field. The direction of in-plane net magnetic moment is represented by the gray arrow. (b) Schematic drawing of the sample device for the electricaltransport measurements used inside the pressure cell.

a nonvanishing Berry curvature that arises from the chiral spin structure. $A b$ initio band structure calculations indicate multiple Weyl points in the bulk band structures of the chiral antiferromagnetic compound $\mathrm{Mn}_{3} \mathrm{Ge}$ with the positions and chirality of Weyl points in accordance with the symmetry of the magnetic lattice $[30,31]$. The discovery of Weyl points verifies that the large anomalous Hall conductivity observed is indeed connected to the electronic structure of the material $[11,32]$.

The exotic transport properties make hexagonal $\mathrm{Mn}_{3} \mathrm{Ge}$ not only interesting for fundamental research but also a promising material for novel applications. $\mathrm{Mn}_{3} \mathrm{Ge}$ belongs to the family of Heusler compounds which is known for their high structural tunability by chemical substitution and external pressure $[11,33,34]$. Although chemical substitution is useful to study the relation between lattice parameters and other physical properties, it also leads to an increase in disorder, which in this case can thwart the exotic transport properties. The use of external pressure, on the other hand, is a powerful approach to continuously tune the physical properties of intermetallic compounds and appears to be the prime choice for controlling the noncollinear triangular antiferromagnetic structure of hexagonal $\mathrm{Mn}_{3} \mathrm{Ge}$.

In this Rapid Communication, we demonstrate that the AHE in hexagonal $\mathrm{Mn}_{3} \mathrm{Ge}$ can be controlled by hydrostatic pressure. Combining our electrical-transport data with previous powder neutron diffraction data allows us to correlate the changes in the AHE amplitude with the gradual change in the magnetic structure. This result demonstrates a way to continuously tune the AHE at room temperature by hydrostatic pressure.

High-quality single crystals of hexagonal $\mathrm{Mn}_{3} \mathrm{Ge}$ were grown using the Bridgman-Stockbarger technique. More details on the sample preparation and ambient pressure characterization can be found elsewhere [8]. The electrical-transport experiments were performed in magnetic fields up to $B=9 \mathrm{~T}$ in a Quantum Design physical property measurement system
(PPMS-9T) at temperatures from room temperature down to $T=5 \mathrm{~K}$. Electrical transport data were recorded in five-point geometry, where the electrical contacts were spot welded to the sample using $25-\mu \mathrm{m}$ platinum wire. The electrical current was applied parallel to the crystallographic [0001] axis and the magnetic field parallel to the [2-1-10] axis. A schematic plot of the electrical-transport experiment setup is displayed in Fig. 1(b). Hydrostatic pressure was generated using a clamptype pressure cell with silicon oil as the pressure-transmitting medium. The pressure inside the cell was determined by the shift of the superconducting critical temperature of a piece of lead placed next to the sample. The narrow width of the superconducting transition at all pressures confirmed the good hydrostatic conditions in the sample chamber.

In order to probe the effect of hydrostatic pressure on the AHE in $\mathrm{Mn}_{3} \mathrm{Ge}$, we carried out detailed electrical-transport measurements as a function of magnetic field for temperatures between 5 and $300 \mathrm{~K}$ at pressures up to $2.85 \mathrm{GPa}$. For that, the sample was cooled in zero magnetic field down to $5 \mathrm{~K}$, and Hall and magnetoresistance were measured in a field loop from -9 to $9 \mathrm{~T}$ and back to $-9 \mathrm{~T}$ again. Consecutively, the next temperatures were approached and loops between -3 and $3 \mathrm{~T}$ recorded. From the Hall and transversal magnetoresistance we calculated the Hall conductivity using the expression $\sigma_{y z}=-\rho_{y z} /\left(\rho_{y z}^{2}+\rho_{z z}^{2}\right)$, where $\rho_{y z}$ and $\rho_{z z}$ are the Hall and transversal resistivity, respectively. We note that the total (observed) Hall conductivity $\sigma_{y z}$ is the sum of the conventional Hall and anomalous Hall conductivities. Figure 2 provides an overview of the pressure effects on the AHE of $\mathrm{Mn}_{3} \mathrm{Ge}$. At ambient pressure, see Fig. 2(a), both $\rho_{y z}(H)$ and $\sigma_{y z}(H)$ abruptly saturate in small magnetic fields of only about $\pm 0.1 \mathrm{~T}$. The saturation value is large, $\left|\sigma_{y z}^{\text {sat }}\right| \approx$ $100 \mu \Omega^{-1} \mathrm{~cm}^{-1}$ at $5 \mathrm{~K}$ and $\left|\sigma_{y z}^{\text {sat }}\right| \approx 40 \mu \Omega^{-1} \mathrm{~cm}^{-1}$ at $300 \mathrm{~K}$. This is in good agreement with previous studies $[8,25]$. At first glance, the large AHE at ambient pressure appears to be due to an antiferromagnetic ordering with a noncollinear spin arrangement that leads to a nonvanishing Berry phase in $\mathrm{Mn}_{3} \mathrm{Ge}$. At $p=2.85 \mathrm{GPa}$, on another side, the highest pressure in our experiment, the amplitude of the Hall signal is inverted with respect to the magnetic field. The data, presented in Fig. 2(b), become almost temperature independent. There is only a small reduction in $\left|\rho_{y z}^{\text {sat }}\right|$ and a small increase in $\left|\sigma_{y z}^{\text {sat }}\right|$ upon decreasing temperature, respectively. The magnitude of $\left|\rho_{y z}^{\text {sat }}\right|$ and $\left|\sigma_{y z}^{\text {sat }}\right|$ is only slightly reduced compared with ambient pressure at $300 \mathrm{~K}$, while there is a much stronger reduction at lower temperatures. In addition, we observe a small hysteresis at $2.85 \mathrm{GPa}$ in the $\rho_{y z}(H)$ and $\sigma_{y z}(H)$ loops whose origin is discussed below.

The most marked feature in $\mathrm{Mn}_{3} \mathrm{Ge}$ is the appearance of the large AHE at room temperature. Therefore, controlling the amplitude of the AHE at room temperature is a key for any future application in devices. In Fig. 3 we show the evolution of $\sigma_{y z}$ as a function of external pressure up to $2.85 \mathrm{GPa}$ at $300 \mathrm{~K}$. The saturation value of $\sigma_{y z}(H)$ can be continuously tuned by increasing pressure from positive to negative values at positive fields and vice versa at negative fields. At $0.05 \mathrm{GPa}$, virtually ambient pressure, we observe a large $\left|\sigma_{y z}^{\text {sat }}\right|$ of $40 \mu \Omega^{-1} \mathrm{~cm}^{-1}$ already for small applied fields. Increasing pressure leads to a dramatic reduction in the saturation value. 

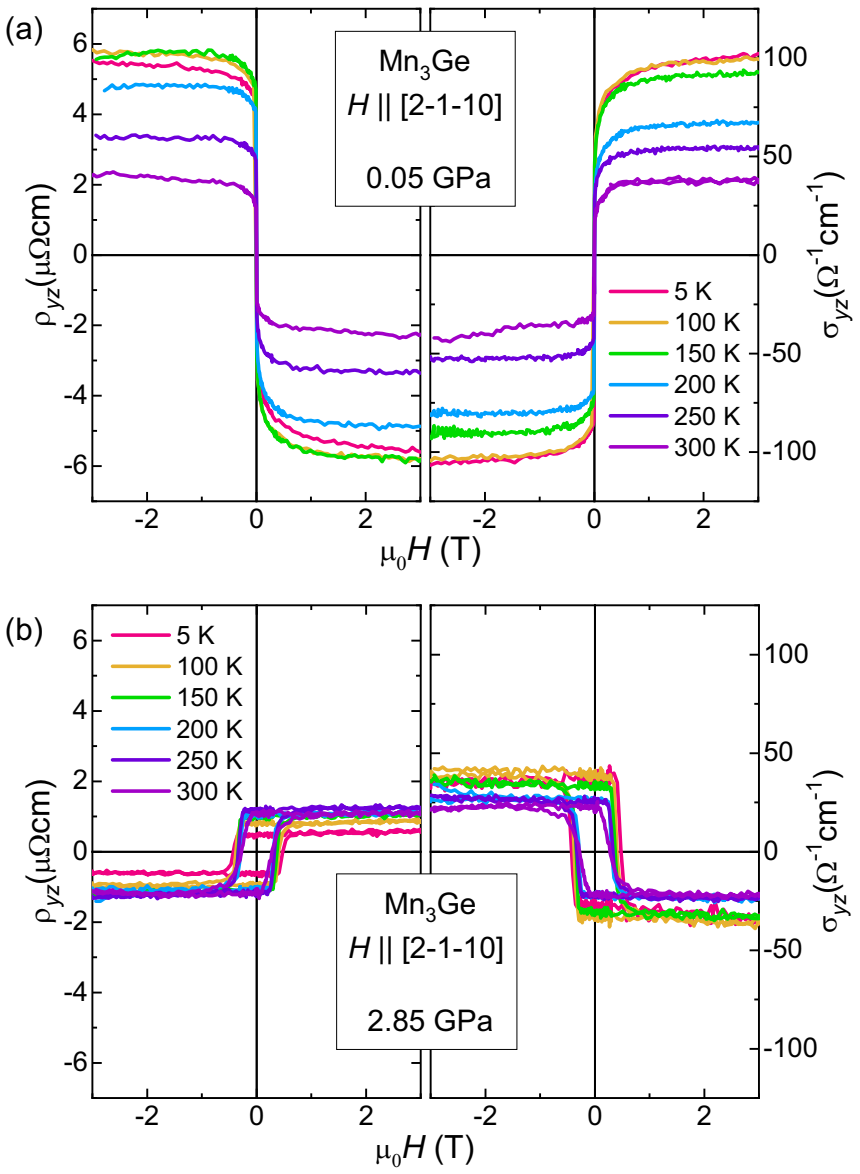

FIG. 2. (a) and (b) Hall resistivity $\left(\rho_{y z}\right)$ and Hall conductivity $\left(\sigma_{y z}\right)$ as a function of magnetic field $(H)$ at representative temperatures between 5 and $300 \mathrm{~K}$ for pressures of 0.05 and $2.85 \mathrm{GPa}$, respectively.

$\sigma_{y z}$ becomes zero in the whole field range at $p=1.53 \mathrm{GPa}$. More interestingly, for higher pressures $\sigma_{y z}$ starts to change sign, reaching a saturation signal $\left|\sigma_{y z}^{\mathrm{sat}}\right|=22 \mu \Omega^{-1} \mathrm{~cm}^{-1}$ at $p=2.85 \mathrm{GPa}$.

At ambient pressure, the large anomalous Hall effect in $\mathrm{Mn}_{3} \mathrm{Ge}$ was discovered before $[8,35]$ and its appearance explained by a nonvanishing Berry curvature due to the noncollinear triangular antiferromagnetic spin structure [22-27]. Our experimental results show a direct correlation between application of hydrostatic pressure and the amplitude of the AHE. As reported by Sukhanov et al. in a neutron diffraction study [20], application of external pressure does not substantially affect the $120^{\circ}$ triangular antiferromagnetic spin structures but induces a dramatic modification of the out-of-plane moment of the spin triangle which can be quantified by the canting angle of the moment with respect to the (0001) plane. Upon increasing pressure the noncollinear triangular magnetic structure of $\mathrm{Mn}_{3} \mathrm{Ge}$ changes gradually to a uniformly canted noncollinear triangular structure and successively to a collinear ferromagnetic structure above $5 \mathrm{GPa}$ [20], which is beyond the pressure range of our present study.

Figure 4(a) illustrates the arrangement of the Mn moments at ambient pressure and at $2.85 \mathrm{GPa}$, the highest pressure of our experiment, clearly indicating the out-of-plane tilting of

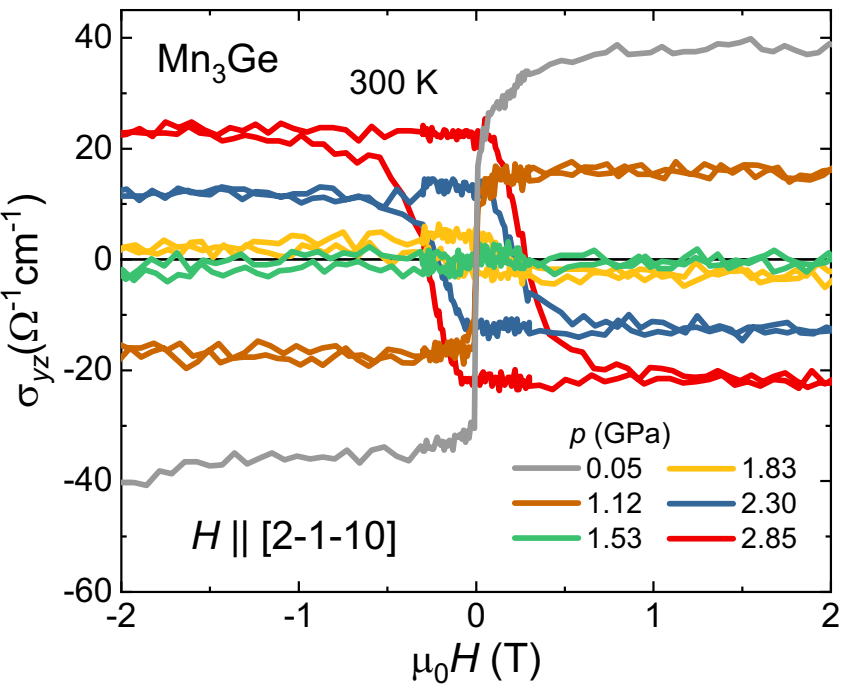

FIG. 3. Field dependence Hall conductivity for $\mathrm{Mn}_{3} \mathrm{Ge}$ at room temperature for selected pressures.

the moments at $2.85 \mathrm{GPa}$. The correlation of the pressureinduced changes in the AHE and the modifications in the magnetic structure of $\mathrm{Mn}_{3} \mathrm{Ge}$ becomes evident in the plot shown in Fig. 4(b). In the temperature-pressure color map the continuous color code corresponds to the size of the jump in the saturation value of the Hall resistivity taken in negative and positive magnetic fields $\Delta \rho_{y z}=2 \times\left|\rho_{y z}^{\text {sat }}\right|$. The canting angle of the Mn moments with respect to the (0001) plane taken from Ref. [20] is indicated by colorized bullets corresponding to the color scheme provided in Fig. 4(b). As a result we find an astonishing correspondence between the jump size of the AHE signal and the canting angle in the whole $T-p$ plane. Thus we can argue that the pressureinduced modifications of AHE are due to modifications of the magnetic arrangement of the Mn moments.

Application of pressure induces a gradual change of the magnetic structure of $\mathrm{Mn}_{3} \mathrm{Ge}$, with the moments starting to move toward the [0001] direction [see Fig. 4(a)]. In our Hall setup, the current is applied along the [0001] direction in parallel with the out-of-plane ferromagnetic moment. Therefore the increasing out-of-plane ferromagnetic moment has no effect on the measured Hall signal. The magnetic configuration in the (0001) plane stays essentially the same but with a reduced size of the in-plane components of the Mn moments.

Beyond the sign change in the amplitude of $\sigma_{y z}$ at around $1.5 \mathrm{GPa}$, the $\sigma_{y z}$ curves start to present a small magnetic hysteresis. The size is slightly increasing with pressure, reaching a "coercive field" of $\mu_{0} H_{c} \approx 0.25 T$. The hysteresis appears due the special spin arrangement present in $\mathrm{Mn}_{3} \mathrm{Ge}$, which leads to a formation of antiferromagnetic domains. At ambient pressure the presence of a weak in-plane ferromagnetic moment ensures that the application of a small magnetic field in the (0001) plane is sufficient to prevent the formation of magnetic domains. However, for higher pressures, with gradual rotation of the spin into [0001] direction, the inplane ferromagnetic moment is reduced, and therefore a larger magnetic field is needed for suppress the antiferromagnetic 
(a)

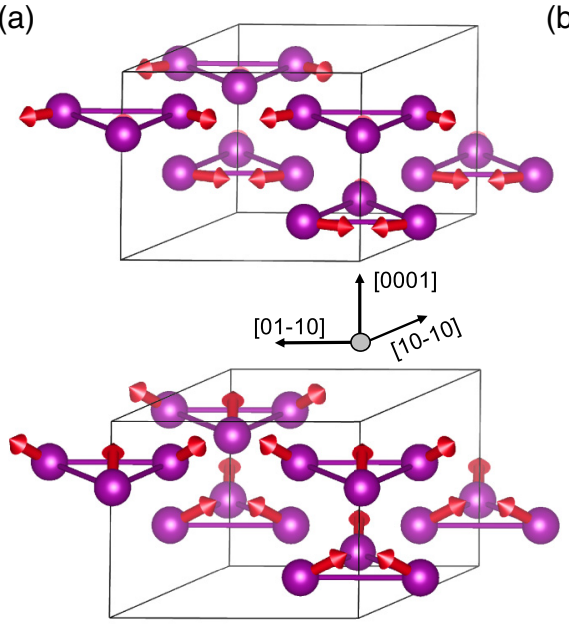

(b)

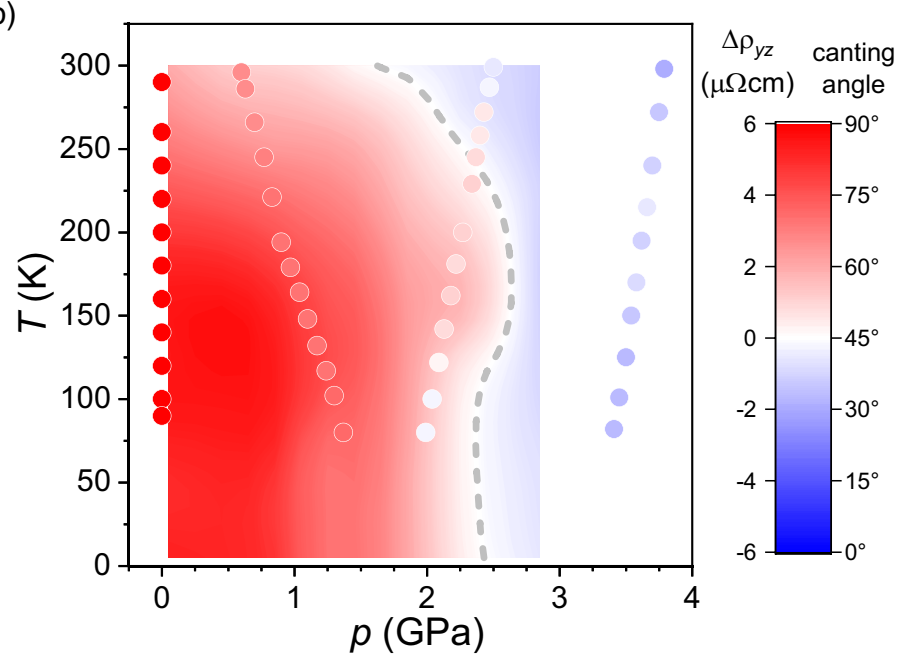

FIG. 4. (a) The inverse triangular magnetic structure of $\mathrm{Mn}_{3} \mathrm{Ge}$ at ambient pressure (top) and at $p=2.85$ GPa (bottom). (b) Pressuretemperature color map of the AHE amplitude $\left(\Delta \rho_{\mathrm{yz}}\right)$ for the $\mathrm{Mn}_{3} \mathrm{Ge}$ compound. The bullets represent the angle of the spin moments out of the (0001) plane extracted from previous neutron diffraction experiments [20,21]. The gray dashed line indicates a vanishing AHE.

domains [20]. We note that although we start to observe a hysteresis in the $\sigma_{y z}$ curves, $\sigma_{y z}$ saturates immediately and stays flat for the entire magnetic field range, indicating that the in-plane ferromagnetic moment plays no role in the observed AHE except in defining the chirality of the spin triangular structure [see Fig. 1(a)].

We have observed that at $300 \mathrm{~K}$ the AHE, $\left|\sigma_{y z}^{\text {sat }}\right| \approx$ $40 \Omega^{-1} \mathrm{~cm}^{-1}$, is continuously suppressed as a function of increasing external pressure. The Hall signal vanishes completely in the full investigated magnetic field range at $p=$ $1.53 \mathrm{GPa}$. With further increasing pressure, the Hall signal changes sign and starts increasing again, reaching a maximum of $\left|\sigma_{y z}^{\text {sat }}\right| \approx 23 \Omega^{-1} \mathrm{~cm}^{-1}$ at $p=2.85 \mathrm{GPa}$. Considering previous powder neutron diffraction results, these data seem to indicate that the observed changes in the AHE are directly connected to modifications in the magnetic configuration. We can then speculate that these are causing changes in the electronic band structure, which further lead to a sign change in the Berry phase. However, density functional theory calculations dealing with $d$ electrons in magnetic systems are challenging in the required precision and make this point an open question for further studies.

In conclusion, we presented an investigation of the pressure evolution of the anomalous Hall effect in the chiral antiferromagnet $\mathrm{Mn}_{3} \mathrm{Ge}$. Our findings demonstrate that application of external pressure can play an efficient role in tuning the anomalous Hall effect in antiferromagnetic materials and offers a route for further exploration of the Berry phase as the physical mechanism behind the AHE.

This work was financially supported by the ERC Advanced Grant No. 742068 “TOP-MAT.” R.D.d.R. and L.O.K. acknowledge financial support from the Brazilian agencies CNPq and FAPESP (Grants No. 2018/00823-0 and No. 2018/19015-1) and from the Max Planck Society under the auspices of the Max Planck Partner Group of the MPI for Chemical Physics of Solids, Dresden, Germany. S.S. acknowledges the Science and Engineering Research Board of India for financial support through an Early Career Research Award (Grant No. ECR/2017/003186) and the award of a Ramanujan Fellowship (Grant No. SB/S2/RJN-015/2017).
[1] K. S. Novoselov, A. K. Geim, S. V. Morozov, D. Jiang, Y. Zhang, S. V. Dubonos, I. V. Grigorieva, and A. A. Firsov, Electric field effect in atomically thin carbon films, Science 306, 666 (2004).

[2] C. Chappert, A. Fert, and F. N. Van Dau, The emergence of spin electronics in data storage, Nat. Mater. 6, 813 (2007).

[3] A. D. Kent and D. C. Worledge, A new spin on magnetic memories, Nat. Nanotechnol. 10, 187 (2015).

[4] M. M. Waldrop, The chips are down for Moore's law, Nature (London) 530, 140 (2016).

[5] E. Liu, Y. Sun, N. Kumar, L. Muechler, A. Sun, L. Jiao, S.-Y. Yang, D. Liu, A. Liang, Q. Xu, J. Kroder, V. Süß, H. Borrmann,
C. Shekhar, Z. Wang, C. Xi, W. Wang, W. Schnelle, S. Wirth, Y. Chen et al., Giant anomalous Hall effect in a ferromagnetic kagome-lattice semimetal, Nat. Phys. 14, 1125 (2018).

[6] S. V. Faleev, Y. Ferrante, J. Jeong, M. G. Samant, B. Jones, and S. S. P. Parkin, Heusler compounds with perpendicular magnetic anisotropy and large tunneling magnetoresistance, Phys. Rev. Mater. 1, 024402 (2017).

[7] T. Jungwirth, J. Sinova, A. Manchon, X. Marti, J. Wunderlich, and C. Felser, The multiple directions of antiferromagnetic spintronics, Nat. Phys. 14, 200 (2018).

[8] A. K. Nayak, J. E. Fischer, Y. Sun, B. Yan, J. Karel, A. C. Komarek, C. Shekhar, N. Kumar, W. Schnelle, J. Kübler, C. 
Felser, and S. S. P. Parkin, Large anomalous Hall effect driven by a nonvanishing Berry curvature in the noncolinear antiferromagnet $\mathrm{Mn}_{3} \mathrm{Ge}$, Sci. Adv. 2, e1501870 (2016).

[9] P. Wadley, B. Howells, J. Železný, C. Andrews, V. Hills, R. P. Campion, V. Novák, K. Olejník, F. Maccherozzi, S. S. Dhesi, S. Y. Martin, T. Wagner, J. Wunderlich, F. Freimuth, Y. Mokrousov, J. Kuneš, J. S. Chauhan, M. J. Grzybowski, A. W. Rushforth, K. W. Edmonds et al., Electrical switching of an antiferromagnet, Science 351, 587 (2016).

[10] X. Hu, Half-metallic antiferromagnet as a prospective material for spintronics, Adv. Mater. 24, 294 (2012).

[11] K. Manna, Y. Sun, L. Muechler, J. Kübler, and C. Felser, Heusler, Weyl and Berry, Nat. Rev. Mater. 3, 244 (2018).

[12] C. Marrows, Addressing an antiferromagnetic memory, Science 351, 558 (2016).

[13] B. G. Park, J. Wunderlich, X. Martí, V. Holý, Y. Kurosaki, M. Yamada, H. Yamamoto, A. Nishide, J. Hayakawa, H. Takahashi, A. B. Shick, and T. Jungwirth, A spin-valve-like magnetoresistance of an antiferromagnet-based tunnel junction, Nat. Mater. 10, 347 (2011).

[14] Y. Ohuchi, J. Matsuno, N. Ogawa, Y. Kozuka, M. Uchida, Y. Tokura, and M. Kawasaki, Electric-field control of anomalous and topological Hall effects in oxide bilayer thin films, Nat. Commun. 9, 213 (2018).

[15] D. Zhang, B. Yan, S.-C. Wu, J. Kübler, G. Kreiner, S. S. P. Parkin, and C. Felser, First-principles study of the structural stability of cubic, tetragonal and hexagonal phases in $\mathrm{Mn}_{3} \mathrm{Z}$ ( $\mathrm{Z}=\mathrm{Ga}$, Sn and Ge) Heusler compounds, J. Phys.: Condens. Matter 25, 206006 (2013).

[16] T. Ohoyama, X-ray and magnetic studies of the manganesegermanium system, J. Phys. Soc. Jpn. 16, 1995 (1961).

[17] J. F. Qian, A. K. Nayak, G. Kreiner, W. Schnelle, and C. Felser, Exchange bias up to room temperature in antiferromagnetic hexagonal $\mathrm{Mn}_{3}$ Ge, J. Phys. D: Appl. Phys. 47, 305001 (2014).

[18] T. Nagamiya, Triangular spin ordering in $\mathrm{Mn}_{3} \mathrm{Sn}$ and $\mathrm{Mn}_{3} \mathrm{Ge}$, J. Phys. Soc. Jpn. 46, 787 (1979).

[19] T. Nagamiya, S. Tomiyoshi, and Y. Yamaguchi, Triangular spin configuration and weak ferromagnetism of $\mathrm{Mn}_{3} \mathrm{Sn}$ and $\mathrm{Mn}_{3} \mathrm{Ge}$, Solid State Commun. 42, 385 (1982).

[20] A. S. Sukhanov, S. Singh, L. Caron, T. Hansen, A. Hoser, V. Kumar, H. Borrmann, A. Fitch, P. Devi, K. Manna, C. Felser, and D. S. Inosov, Gradual pressure-induced change in the magnetic structure of the noncollinear antiferromagnet $\mathrm{Mn}_{3} \mathrm{Ge}$, Phys. Rev. B 97, 214402 (2018).

[21] J.-R. Soh, F. de Juan, N. Qureshi, H. Jacobsen, H.-Y. Wang, Y.F. Guo, and A. T. Boothroyd, Ground-state magnetic structure of $\mathrm{Mn}_{3} \mathrm{Ge}$, Phys. Rev. B 101, 140411(R) (2020).
[22] J. Kübler and C. Felser, Non-collinear antiferromagnets and the anomalous Hall effect, Europhys. Lett. 108, 67001 (2014).

[23] H. Chen, Q. Niu, and A. H. MacDonald, Anomalous Hall Effect Arising from Noncollinear Antiferromagnetism, Phys. Rev. Lett. 112, 017205 (2014).

[24] R. Shindou and N. Nagaosa, Orbital Ferromagnetism and Anomalous Hall Effect in Antiferromagnets on the Distorted fcc Lattice, Phys. Rev. Lett. 87, 116801 (2001).

[25] S. Nakatsuji, N. Kiyohara, and T. Higo, Large anomalous Hall effect in a non-collinear antiferromagnet at room temperature, Nature (London) 527, 212 (2015).

[26] Y. Zhang, Y. Sun, H. Yang, J. Železný, S. P. P. Parkin, C. Felser, and B. Yan, Strong anisotropic anomalous Hall effect and spin Hall effect in the chiral antiferromagnetic compounds $\mathrm{Mn}_{3} X$ ( $X=\mathrm{Ge}, \mathrm{Sn}, \mathrm{Ga}$, Ir, Rh, and Pt), Phys. Rev. B 95, 075128 (2017).

[27] Y. Zhang, J. Železný, Y. Sun, J. van den Brink, and B. Yan, Spin Hall effect emerging from a noncollinear magnetic lattice without spin-orbit coupling, New J. Phys. 20, 073028 (2018).

[28] Y. Taguchi, Y. Oohara, H. Yoshizawa, N. Nagaosa, and Y. Tokura, Spin chirality, Berry phase, and anomalous Hall effect in a frustrated ferromagnet, Science 291, 2573 (2001).

[29] N. J. Ghimire, A. S. Botana, J. S. Jiang, J. Zhang, Y. S. Chen, and J. F. Mitchell, Large anomalous Hall effect in the chiral-lattice antiferromagnet $\mathrm{CoNb}_{3} \mathrm{~S}_{6}$, Nat. Commun. 9, 3280 (2018).

[30] H. Yang, Y. Sun, Y. Zhang, W.-J. Shi, S. S. P. Parkin, and B. Yan, Topological Weyl semimetals in the chiral antiferromagnetic materials $\mathrm{Mn}_{3} \mathrm{Ge}$ and $\mathrm{Mn}_{3} \mathrm{Sn}$, New J. Phys. 19, 015008 (2017).

[31] J. Kübler and C. Felser, Weyl fermions in antiferromagnetic $\mathrm{Mn}_{3} \mathrm{Sn}$ and $\mathrm{Mn}_{3} \mathrm{Ge}$, Europhys. Lett. 120, 47002 (2017).

[32] C. Wuttke, F. Caglieris, S. Sykora, F. Scaravaggi, A. U. B. Wolter, K. Manna, V. Süss, C. Shekhar, C. Felser, B. Büchner, and C. Hess, Berry curvature unravelled by the anomalous Nernst effect in $\mathrm{Mn}_{3} \mathrm{Ge}$, Phys. Rev. B 100, 085111 (2019).

[33] F. Casper, T. Graf, S. Chadov, B. Balke, and C. Felser, Half-Heusler compounds: Novel materials for energy and spintronic applications, Semicond. Sci. Technol. 27, 063001 (2012).

[34] T. Graf, S. S. P. Parkin, and C. Felser, Heusler compounds-A material class with exceptional properties, IEEE Trans. Magn. 47, 367 (2011).

[35] N. Kiyohara, T. Tomita, and S. Nakatsuji, Giant Anomalous Hall Effect in the Chiral Antiferromagnet $\mathrm{Mn}_{3} \mathrm{Ge}$, Phys. Rev. Appl. 5, 064009 (2016). 